\title{
SOCIAL METACOGNITION IN THE PROCESS OF DECISION MAKING
}

\author{
Tatiana V. Folomeeva, \& Ekaterina N. Klimochkina \\ Department of Psychology, Lomonosov Moscow State University (Russia)
}

\begin{abstract}
Individual metacognitions are responsible for monitoring and controlling our knowledge, emotions and actions, while social metacognitions are included in the process of monitoring and controlling each other's knowledge, emotions and actions by group members. The distribution of metacognitive responsibilities among group members increases the visibility of individual metacognitive abilities. The study aimed to investigate the role of social metacognitions in the decision process of choosing current fashion trends: to compare how participants interact and social metacognitions influence their decisions in contradiction to the decisions made in individual work, where only individual metacognitions were available. The study consisted of several stages: starting from current trends analysis and follow up interviews, as well as, filling in individual journals. The last step was group work: discussion. The sample was 40 participants $(M=24,35$, $\mathrm{Sd}=2,27)$. Gathered data was processed through a descriptive qualitative analysis using the phenomenological method. Lack of knowledge or confidence to make a decision about which trends represent what is current in fashion in individual work, participants compensate with knowing about their own metacognition. Thus, in teamwork, these individuals' level out limitations on knowledge or confidence by choosing the behaviour that can increase their knowledge. Choice of the behaviour strategy relies on individual metacognition. Therefore, teamwork provides individuals with additional resources as other team members, which increases the overall significance of work due to the contribution of individual metacognition. Social metacognitions help to distribute responsibilities among group members according to individual metacognitions. In group work, the visibility of individual metacognitions increases and favourably affects learning between participants, facilitates interaction and improves cognitive processes. Due to social metacognitions, participants who lack knowledge or confidence to make an individual decision solve their difficulties in a social situation, where limitations of individual metacognition are mitigated with social metacognitions.
\end{abstract}

Keywords: Metacognition, social metacognition, fashion trends, metacognitive awareness, metacognitive beliefs.

\section{Introduction}

We monitor and control our own cognitive processes and this process refers to metacognition. It can be divided into types, implicit and explicit forms, where implicit means automatic and unconscious. We automatically take into account the knowledge and intentions of others through implicit metacognition and adopt "we" mode, which strengthens joint actions. We reflect and justify our behaviour to others through explicit metacognition. However, access to this level is limited for both ourselves and others and our reports on the intentions of others or ourselves can be inaccurate (Frith, 2012).

Research has shown that by discussing our experiences with others, we can recognize them more accurately, even in the absence of objective feedback, as an example, perceptual experiences and sensory cues (Wolfe, 2018). Thus, it creates the potential for us to build accurate knowledge about ourselves and the world we live in. Therefore, the ability to share our values and beliefs, to have a common view of the world is provided by explicit metacognition (Friston, 2015). Explicit metacognition is a uniquely human ability that has evolved through enhanced collaborative decision-making.

One of the central metacognitive assessments that people make is the confidence they attach to their judgments and decision-making. People tend to act decisively based on those judgments in which they are most confident. People are more likely to act cautiously and consult others when they come to judgments they are unsure of. Due to the process of decision making having confirmation from others can reduce the need to find additional data to support the cost of a decision. Collective agreement leads to the accuracy of decision despite a lack of feedback or individual confidence (Bahrami, 2016). Similarly, concurring opinions reduce uncertainty and related information that is compatible with the previous solution which 
also increases confidence. Desire to increase confidence despite costs appears like a result of motivation, Schwarz suggests that individuals who are not motivated to process information often base their judgment on how they retrieve information, whereas the motivated are likely to base their judgment on what they retrieve. Therefore, the base for judgments is not only in what comes to mind but also in how comes to mind.

Confidence in the judgement is formed based on previous responses and feedback, which can only happen after a metacognitive assessment of social sources. Shared responsibility for decisions helps to justify decisions and choices (Chiu, 2009). To test confidence people rely on the process of comparing themselves to others or other versions of themselves (past or future). Confidence and satisfaction are key indicators of metacognition. Individual metacognition stands for awareness and control of one's own knowledge, emotions, and actions. Social metacognition is the process responsible for monitoring and controlling each other's knowledge, emotions, and behaviour by group members, and making individual metacognition visible to enhance each group member's experience (Chiu, 2009). Therefore, social metacognition conveys metacognitive requirements among members of the group to increase the visibility of each other's metacognition for the development and enhancement of individual cognition (Carlson, 2016).

Spread responsibility enhances the visibility of metacognitive processes and improves individual cognition. When in teamwork there is an offer to express ideas to each other's or share own thoughts, members of the group must explicitly communicate through words, actions, facial expressions, and etc. (Dillenbourg \& Traum, 2006). In collective activities, individuals focus more confidently on cognitive processes and assume responsibility for some metacognitive roles. Greater visibility of cognitive and metacognitive processes also facilitates metacognitive assessment for recognizing correct ideas and easier process of making decisions (Hurme et. al., 2006). In the act of expressing an idea, group members can explore explicit ideas more thoroughly, especially to recognize problems or difficulties of a given idea. Therefore, the ability to share our values and beliefs, to have a common view of the world, where useful group communication is provided, is provided by explicit metacognition as well (Friston, 2015).

Beliefs, values, and goals mostly depend on heritage, family system, and group membership, and the surrounding context. Therefore, metacognitive beliefs can be experimentally altered by social influence (Dweck, 1990). Immersion in society causes interference between the individual and the social. Studies have highlighted the role of metacognitive judgments in social interactions, as well as whether social context can mutually influence individuals ' metacognitive perceptions (Chiu \& Kuo, 2009). Thus, individuals, as a part of society, can interact, along with exposure to external influences. Metacognitive beliefs are the part of the decision-making process and the product of attitudes, stereotypes and culture (Wells, 2000). The change of metacognitive beliefs is impacted by the received information as soon as it differs from previous beliefs of the individual. We depend on metacognitive beliefs in the measurement of our level of satisfaction with our decisions (Wolfe, 2018).

\section{Objectives}

The research aimed to investigate the role of social metacognition in the process of decision-making. We hypothesized, that level of confidence in personal decisions (upon fashion trends and relevance in fashion) corresponds with behavioural strategy in social interactions and correlates with metacognitive beliefs.

Metacognitions, specifically, social metacognitions impact individual's awareness and control over cognitive processes, self-regulation of emotions, actions, knowledge in a group activity, therefore, metacognitions and social metacognitions interfere with the way individual's decision-making and stays confident in it.

\section{Methods and design}

The sample consisted of 40 participants (young females and males (first stage $\mathrm{M}=27,7, \mathrm{Sd}=3,6$; second stage $\mathrm{M}=21, \mathrm{Sd}=0,94$ ), concluded of university students and experts in the fashion field (following fashion trends, involvement in fashion subcultures, professionals of the fashion field).

The research consisted of several stages. We began by collecting data about relevant fashion trends through current trend-books of marketing agencies (McKinsey\&Co).

Thereupon, to investigate a unique understanding of fashion trends 15 interviews with fashion experts were arranged. Gathered qualitative data was processed through a descriptive qualitative analysis using the phenomenological method, where central categories among participants answers were defined.

The second stage of the study consisted of filling in the journal by a control group (25). The journal was developed specifically for the research to study participants daily routine, significant values and fashion 
interests. The journal consisted of 36 pages, which included instructions, an introduction with an informed consent form, as well as, various daily categories to fill in, such as mood chart, activity chart, highlights of the day, day satisfaction rate and forms to collect "fashion" data: what fashion trends do you prefer, what is your dream purchase, what your ideal self looks like. Participants were asked to complete 10 days of journaling. At the end of this period, participants were involved in a focus-group, equally, to highlight the experiences of the participants, as well as, to clarify the given answers.

The third part of the research aimed to investigate group work, participants were working on a creative task together. They were asked to reflect on the journaling experience in a form of creating a collective collage in mini-groups, where the roles of creators and analysts (who monitored and recorded steps of the work) were distributed naturally. Participants were presenting their creations in teams. Afterwards, participants were asked to analyse the collages and process of group work.

Alongside, all 40 participants filled in questionnaires to assess the specificity of metacognitions quantitatively. The Metacognitive Awareness Inventory questionnaire (MAI) (Schraw \& Dennison, 1994) in the adaptation (Karpov \& Skiteva, 2005), allowed to measure the level of metacognitive involvement. Short Form of Metacognitions Questionnaire: Properties of MCQ-30 (Wells \& Cartwright-Hatton, 2004) with the approbation on the Russian-speaking population (Sirota et.al., 2018) provided data about techniques of control and regulation in the information processing at the meta-level.

\section{Discussion}

In accordance with the analysis of the trend book for 2021, we discovered relevant fashion trends (both messages, concepts and clothing):

- Living with the virus (economic crisis and fashion bounce back)

- Consumer shifts to digital (growth of online businesses), the expectancy of fashion brands to care for employees and customers health, travel interruption

- Fashion system: less is more, loyalty and deeper partnerships with meaningful businesses, market redistribution, new norms for working models

Fashion experts who participated in the research clarified the importance of discovers fashion trends, which allowed us to compare further results to a found standard. Gathered through interviews quality data covers a variety of questions, however, we aimed to investigate the process, rather than the context.

Moving to the results of a control group, we discovered through journals analysis, that participants tend to value several concepts dedicated to life and fashion:

- Lower significance of fashion

- Preference to the online activities

- Less is more: recycling, thrifting, quality over everything else

- Being loyal to approved brands and places, as well as, friends and family

- Self-care: priority to health, body positivity, working out, clean eating, self-acceptance

- New norms: live for yourself and dress for yourself, simplicity

- Femininity (breathy clothing, nude colours, romance)

We need to point out that the freedom of the journaling format created a gap between participants: some used this research to their advantage and fully cooperated, while some filled in the journal briefly. Thus, the difference in "filling level" corresponds with individual results. Correspondently, those who filled in a journal shortly rated day satisfaction the lowest and had a higher significance of metacognitive beliefs (see Fig.1). To illustrate, one of the participants rated the day 5,6 on average with 8,64 variance and had several metacognitive beliefs (positive beliefs about worry, uncontrollability/danger belief, cognitive confidence, cognitive self-consciousness). Using A. Wells theory of metacognitive beliefs, we can state, that this participant worries, feels anxious, catastrophizes the obstacles, experiences uncontrollability and has decreased cognitive functioning. Also, analysis of this participant's journal shows that after finishing certain tasks (participant was asked to create a stylish look) dedicated to fashion knowledge, a participant was happy with the finished result and self-rated it at the highest mark, but later on, there was an opportunity to save or modify the look which participant rejected. As a next step, we asked (one of the written questions) participant to rate the "created look" afterwards, which led to doubts, lower second self-rating of the task and a request to change it. Therefore, this participant showed a change in confidence due to the assessment of an answer. This example is taken to illustrate the inner and outer behaviour of participants who gained several metacognitive beliefs and overall were less satisfied daily (with their life, as well as, with the research). The same algorithm happened with several participants on various questions about fashion knowledge. 


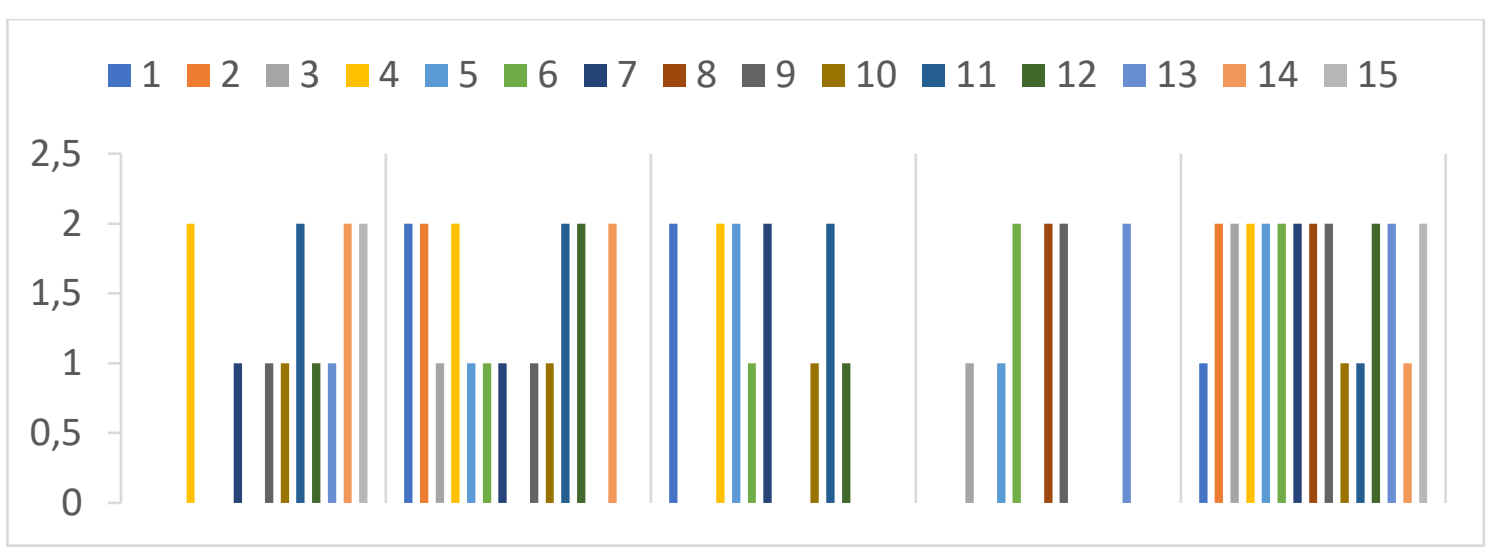

After individual work with journals, we brought participants together and asked them to create collages for the same topic "Relevant state of fashion". Participants were allocated in mini-groups of 3 to 4 with naturally occurred roles of creators and analysts. Coincidently, the participant from the previous example took an analyst role, which consisted of monitoring actions and ideas of creators and summing a discussion in the group. Analysts of mini-groups were asked to make a report afterwards with an analysis of their group's collages and all groups' artworks to give an overview of relevant fashion trends. Accordingly, a participant from the example did an amazing job, made a detailed report with substantial details of team members opinions and arguments to prove what and why is relevant in fashion.

The difference in poor individual work (due to metacognitive beliefs, lack of knowledge or else) and in the report after group work we identify as one's social metacognition. The participant felt not enough knowledge, nor confidence to rate "created look" successfully, therefore, had a negative judgement on own work known as metacognitive accuracy. In teamwork, participant used metacognitive knowledge about oneself (I'm not knowledgable in fashion) and decided to be the analyst in a creative group task. By deciding on this role participant demonstrated self-regulation (I'll better watch and learn, rather than fail as a creator). The decision of an adequate role gave an ability to learn and perform a successful report.

The process of listening to teammates opinions helped to gain knowledge, just like, observing and monitoring each other as a team. Natural role occurring created a situation where each group member showcased personal strength and highlighted individual metacognitions. Together they divided responsibilities: who has better knowledge in fashion, who is better at finding relevant pictures in magazines, etc. Collectively they rejected irrelevant information and reassembled the collage. They did it in a form of a discussion, where they shared ideas and expanded individual opinions on spheres of disagreement (which was protocoled by the analyst). By working as a team each group member exhibited individual strength and made individual metacognitions visible, thus, others used this knowledge to impact collective work and made easier and better decisions. Some participants showed better knowledge of clothing, others in concepts, some were not interested in the topic overall, but in the end, all of them completed the task successfully.

Social metacognition is the process of monitoring each other's knowledge, sustaining emotional wellbeing, using one's strengths as a visible enhancer to individual metacognition led to each group member's increased experience, which led to a peaceful, confident and justified decision on what is relevant in fashion. Therefore, as a result of collective work and decision-making a list of valued fashion and life concepts was broadened in comparison to individual work and consisted of:

- Self-worth: self-care and health routines

- Easy-living: second life of things, being "green" and caring towards each other and nature

- Financial stability: economics and role of being able to work and earn (online, for instance)

- Lower significance of what is considered "worthy"

- Physical appearance (feminine, cosy, nostalgic)

\section{Conclusion}

Individual work leads to benefits thoroughly from individual metacognition, which affects accuracy and confidence in the process of decision making. If an individual has metacognitive beliefs, such as positive beliefs about danger, uncontrollability/danger belief, cognitive confidence, cognitive self-consciousness, therefore, an individual is affected by it and lacks abilities to perform at one's own best. Meaning, individual considers himself as not knowledgeable or not competent. Relying on personal 
experience, there are ways to use individual metacognitive knowledge and solve problems, manage the situation or make a decision.

However, the limits of one individual provide a plethora of extra resources in a form of others. In the process of decision making what fashion trends are relevant, collective agreement leads to the accuracy of decision despite lack of knowledge or confidence, which was shown individually in journaling at first. Teamwork provides additional resources as other team members. Spreading tasks between the group increases the visibility of others metacognition, thus, increases the overall significance of work due to the contribution of individual metacognitions. Social metacognitions help to distribute responsibilities among group members according to individual metacognitions. Due to social metacognitions, participants who lack knowledge or confidence to make an individual decision solve their difficulties in a social situation, where limitations of individual metacognitions are mitigated with social metacognitions.

\section{References}

Bahrami, B., Pescetelli, N., \& Rees, G. (2016). The Perceptual and Social Components of Metacognition. Journal of Experimental Psychology, 145(8), 949-965.

Carlson, E. (2016). Meta-Accuracy and Relationship Quality: Weighing the Costs and Benefits of Knowing What People Really Think About You. Journal of Personality \& Social Psychology, 111(2), 250-264.

Chiu, M. (2009). Social metacognition in groups: Benefits, difficulties, learning, and teaching. Journal of Education Research, 3(4), 1-19.

Chiu, M., \& Wing Kuo, S. (2009). From Metacognition to Social Metacognition: Similarities, Differences, And Learning. Journal of Education Research, 1-19.

Dillenbourg, P., \& Traum, D. (2006). Sharing solutions: Persistence and grounding in multimodal collaborative problem solving. Journal of the Learning Sciences, 15(1), 121-151.

Dweck, C. (1990). Self-theories and goals: Their role in motivation, personality, and development. Current theory and research in motivation, 199-235.

Folomeeva, T., \& Klimochkina, E. (2019). Features of Metacognition and Perception of Fashion Trends Among Youth. The European Proceedings of Social \& Behavioural Sciences, 10.15405.

Friston K., F. C. (2015). A Duet for one. Consciousness and Cognition, 390-405.

Frith, C. (2012). The role of metacognition in human social interactions. The Royal Society Publishing, 367(1599), https://doi.org/10.1098/rstb.2012.0123.

Gajdos, T., Regner, I., \& Huguet, P. (2019). Does social context impact metacognition? Evidence from stereotype threat in a visual search task. PLOS, 10.1371/journal.pone. 0215050.

Hacker, D., \& Bol, L. (2004). Metacognitive theory: Considering the social-cognitive influences. In D. M. Etten. Greenwich, Connecticut: Information Age Publishing, Inc.

Hurme, T. R., Palonen, T. and J rvel , S. (2006). Metacognition in joint discussions: An analysis of the patterns of interaction and the metacognitive content of the networked discussions in mathematics. Metacognition Learning, 1, 181-200.

Schraw, G., \& Dennison, R. (1994). Assesing Metacognitive Awareness. Contemporary educational psychology, 19, 460-475.

Sirota, N., Moskovchenko, D., Yaltonsky, V., \& Yaltonskaya, A. (2018). Approbation of the Short Version Questionnaire Metacognitive Belief in Russian-Speaking Population. Psychology. Journal of the Higher School of Economics, 15(2), 307-325.

Wells, A. (2000). Emotional Disorders and Metacognition: Innovative Cognitive Therapy. UK: Wiley: Chichester.

Wolfe, W. (2018). Poor metacognitive awareness of belief change. Quarterly Journal of Experimental Psychology, 71(9), 1898-1910. 